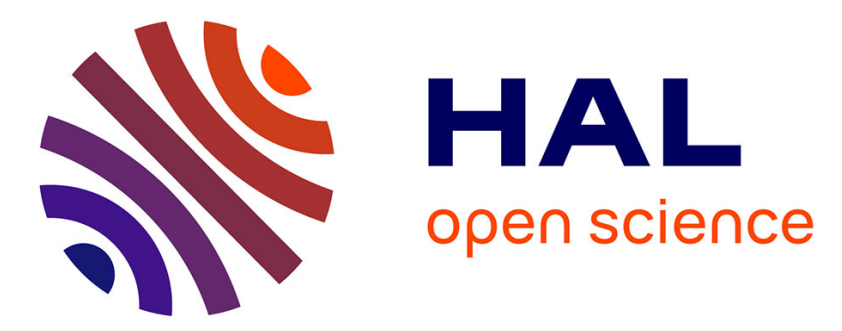

\title{
Microstrain-level measurement of third-order elastic constants applying dynamic acousto-elastic testing
}

\author{
Guillaume Renaud, Maryline Talmant, Guillaume Marrelec
}

\section{To cite this version:}

Guillaume Renaud, Maryline Talmant, Guillaume Marrelec. Microstrain-level measurement of thirdorder elastic constants applying dynamic acousto-elastic testing. Journal of Applied Physics, 2016, 120 (13), pp.135102. 10.1063/1.4963829 . hal-01379025

\section{HAL Id: hal-01379025 \\ https://hal.sorbonne-universite.fr/hal-01379025}

Submitted on 11 Oct 2016

HAL is a multi-disciplinary open access archive for the deposit and dissemination of scientific research documents, whether they are published or not. The documents may come from teaching and research institutions in France or abroad, or from public or private research centers.
L'archive ouverte pluridisciplinaire HAL, est destinée au dépôt et à la diffusion de documents scientifiques de niveau recherche, publiés ou non, émanant des établissements d'enseignement et de recherche français ou étrangers, des laboratoires publics ou privés. 


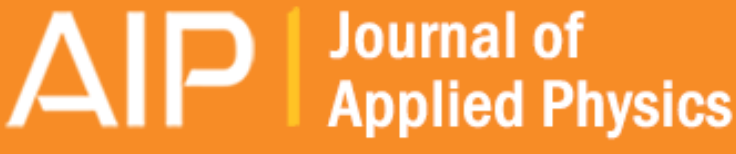

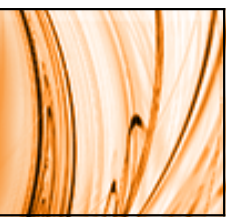

\section{Microstrain-level measurement of third-order elastic constants applying dynamic}

\section{acousto-elastic testing}

Guillaume Renaud, Maryline Talmant, and Guillaume Marrelec

Citation: Journal of Applied Physics 120, 135102 (2016); doi: 10.1063/1.4963829

View online: http://dx.doi.org/10.1063/1.4963829

View Table of Contents: http://scitation.aip.org/content/aip/journal/jap/120/13?ver=pdfcov

Published by the AIP Publishing

\section{Articles you may be interested in}

Third- and fourth-order constants of incompressible soft solids and the acousto-elastic effect

J. Acoust. Soc. Am. 127, 2759 (2010); 10.1121/1.3372624

Determination of third order elastic constants in a complex solid applying coda wave interferometry Appl. Phys. Lett. 94, 011904 (2009); 10.1063/1.3064129

AN ULTRASONIC ANGLE BEAM METHOD FOR DETERMINING THIRD ORDER ELASTIC CONSTANTS VIA ACOUSTOELASTICITY MEASUREMENTS

AIP Conf. Proc. 975, 1207 (2008); 10.1063/1.2902570

Laser ultrasonic determination of the elastic constants of damaged propellant

AIP Conf. Proc. 615, 1392 (2002); 10.1063/1.1472957

Determination of the higher-order elastic compliance constants of metals from measurements of the dependence of ultrasound velocity on stress

J. Acoust. Soc. Am. 101, 2111 (1997); 10.1121/1.418142

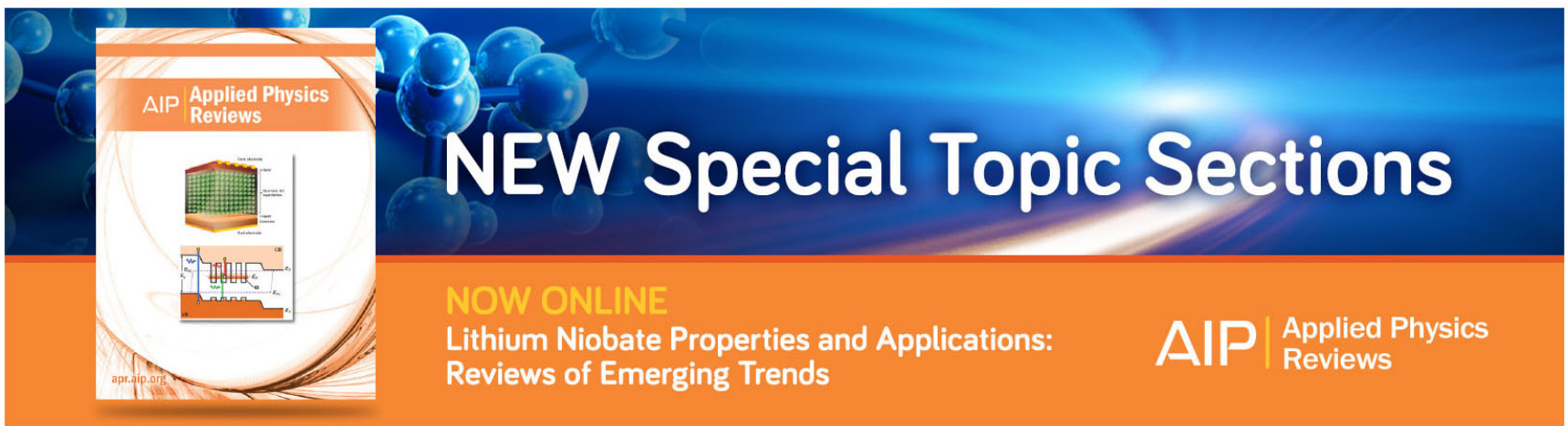




\title{
Microstrain-level measurement of third-order elastic constants applying dynamic acousto-elastic testing
}

\author{
Guillaume Renaud, ${ }^{\text {a) }}$ Maryline Talmant, and Guillaume Marrelec \\ Sorbonne Universités, UPMC Univ Paris 06, CNRS UMR 7371, INSERM UMR S 1146, Laboratoire \\ d'Imagerie Biomédicale, 15 rue de l'école de médecine, 75006 Paris, France
}

(Received 11 June 2016; accepted 18 September 2016; published online 3 October 2016)

\begin{abstract}
The nonlinear elasticity of solids at the microstrain level has been recently studied by applying dynamic acousto-elastic testing. It is the analog of conventional quasi-static acousto-elastic experiments but the strain-dependence (or stress-dependence) of ultrasonic wave-speed is measured with an applied strain ranging from $10^{-7}$ to $10^{-5}$ and produced by a stationary elastic wave. In conventional quasistatic acousto-elastic experiments, the strain is applied in a quasi-static manner; it exceeds $10^{-4}$ and can reach $10^{-2}$. In this work, we apply dynamic acousto-elastic testing to measure the third-order elastic constants of two isotropic materials: polymethyl methacrylate and dry Berea sandstone. The peak amplitude of the dynamic applied strain is $8 \times 10^{-6}$. The method is shown to be particularly suitable for materials exhibiting large elastic nonlinearity like sandstones, since the measurement is performed in the domain of validity of the third-order hyperelastic model. In contrast, conventional quasi-static acousto-elastic experiments in such materials are often performed outside the domain of validity of the third-order hyperelastic model and the stress-dependence of the ultrasonic wave-speed must be extrapolated at zero stress, leading to approximate values of the third-order elastic constants. The uncertainty of the evaluation of the third-order elastic constants is assessed by repeating multiple times the measurements and with Monte-Carlo simulations. The obtained values of the Murnaghan third-order elastic constants are $l=-73 \mathrm{GPa} \pm 9 \%, m=-34 \mathrm{GPa} \pm 9 \%$, and $n=-61 \mathrm{GPa} \pm 10 \%$ for polymethyl methacrylate, and $l=-17000 \mathrm{GPa} \pm 20 \%, m=-11000 \mathrm{GPa} \pm 10 \%$, and $n=-30000 \mathrm{GPa} \pm 20 \%$ for dry Berea sandstone. Published by AIP Publishing. [http://dx.doi.org/10.1063/1.4963829]
\end{abstract}

\section{INTRODUCTION}

The third-order elastic constants (TOECs) evaluate the first-order deviation from linear elasticity of solid materials. ${ }^{1-3}$ The measurement of the TOECs is of interest for the validation of models describing the thermo-elastic behavior of crystals and phonon-phonon interactions. ${ }^{1,2}$ Prior-knowledge of the TOECs for a given material was proposed to be used for the assessment of internal stress in metals ${ }^{4,5}$ and in rocks. ${ }^{6}$ Several methods have been proposed to measure the TOECs: ultrasonic harmonic generation of a single finite amplitude wave and ultrasonic two-wave nonlinear mixing, ${ }^{7}$ shock wave compression, ${ }^{8,9}$ and stress-dependence of ultrasonic wavespeed (or acousto-elasticity). ${ }^{10}$ Methods based on the measurement of the acousto-elastic effect have been used the most to determine the complete set of independent TOECs. The task is rather straightforward in isotropic materials (3 independent TOECs $)^{11}$ but becomes arduous in anisotropic materials (e.g., cubic crystals have 6 independent TOECs ${ }^{12}$ and orthorhombic crystals have 20 independent TOECs ${ }^{13}$ ). The reason why acousto-elastic methods were used the most comes likely from the fact that the observed phenomenon (stress-dependence of ultrasonic wave-speed) is more easily measured. The maximum applied stress (typically hydrostatic or uniaxial) can be adapted to the sensitivity of the technique that monitors the change of wave-speed (as long as the stress does not induce plastic deformation). Conventional acousto-elastic

a)guillaume.renaud@upmc.fr experiments are quasi-static, the applied stress is varied step by step, and ultrasonic wave-speed is measured for each value of the applied stress. ${ }^{11,12}$ While early conventional quasistatic acousto-elastic measurements employed a static loading up to $1 \mathrm{GPa},{ }^{11,14,15}$ recent studies have used less than $10 \mathrm{MPa} .{ }^{16}$ The method to monitor the change of wave-speed induced by the quasi-static loading can be ultrasonic (propagating short burst ${ }^{14}$ or resonance technique ${ }^{17}$ ) or hybrid optical and ultrasonic (diffraction of light by standing elastic wave $^{13}$ or Brillouin spectroscopy ${ }^{18}$ ).

Perhaps surprisingly, literature from the 1980s reports very little work on the measurement of the complete set of TOECs in solids. Recent theoretical work on the ab initio calculation of TOECs refers to the measurements performed in the 1960 s or 1970 s. ${ }^{19}$ While it is generally accepted that the TOECs are difficult to measure experimentally, ${ }^{19}$ very little work has been dedicated to the improvement of the accuracy and precision of the acousto-elastic measurement of the TOECs in the last three decades. However, significant work was published in the past three decades on the development of ways to measure the acousto-elastic effect in solids, without the objective of evaluating the complete set of TOECs though. The quasi-static applied stress was proposed to be replaced with a slowly varying loading. ${ }^{20,21}$ Techniques relying on the interaction between two bulk elastic waves ${ }^{22}$ or surface waves ${ }^{23,24}$ were also studied.

Dynamic Acousto-Elastic Testing (DAET) ${ }^{25-29}$ belongs to this last family of methods. It is the dynamic analog of a conventional quasi-static acousto-elastic measurement. However, 
there exist differences between the DAET and the conventional approach to measure the acousto-elastic effect. First, the applied loading is not quasi-static but induced by a stationary elastic wave. In a typical lab measurement, the stationary elastic wave has a frequency of a few $\mathrm{kHz}$. This means that the elastic constants at stake are all adiabatic elastic constants, unlike a quasi-static acousto-elastic measurement where both adiabatic and isothermal elastic constants are involved. ${ }^{10}$ Second, it can be applied at low vibrational strain, typically $10^{-6}$, while conventional quasistatic acousto-elastic measurements operate with an applied strain exceeding $10^{-4}$. Finally DAET explores the dynamic elastic behavior about the equilibrium state of the material, i.e., both tensile and compressive behaviors are investigated, while quasi-static experiments apply a compressive stress only (or tensile stress only). Although DAET was applied to the investigation of nonlinear elasticity in various media, it was not used to measure the complete set of TOECs of a given material.

In this study, we applied DAET to the measurement of the three independent TOECs of two isotropic materials (polymethyl methacrylate (PMMA) and dry Berea sandstone) at a low strain level, namely, $8 \times 10^{-6}$. The choice of these two materials was motivated by the fact that their nonlinear elastic behaviors differ quantitatively and qualitatively. PMMA is a standard polymer material exhibiting small and simple elastic nonlinearity; it is useful as a reference material in order to validate the experimental method. Berea sandstone is a consolidated granular material with high elastic nonlinearity that was widely studied by geoscientists. To our knowledge, Winkler and McGowan ${ }^{16}$ reported the conventional quasi-static acousto-elastic measurements in PMMA and Berea sandstone performed with the smallest applied strain, namely, of order $10^{-4}$ (i.e., an applied stress of a few MPa). Therefore, we compared their results to those obtained in this study applying dynamic acousto-elastic testing.

After recalling the principles of dynamic acousto-elastic testing in Section II A, the relations between the change of ultrasonic wave-speed and the TOECs are given in Section II B. Then, the experiments are described in Section IIC. Section II D is dedicated to the estimation of the TOECs and their uncertainty. In Section III, we compare our results to the literature and discuss different causes that may explain the discrepancies observed between DAET and conventional quasi-static acousto-elastic measurements.

\section{MEASUREMENT OF THIRD-ORDER ELASTIC CONSTANTS (TOECS) APPLYING DYNAMIC ACOUSTO-ELASTIC TESTING}

\section{A. Principles of dynamic acousto-elastic testing}

A detailed description of the technique can be found in Refs. 30 and 31 . We recall here only what is essential for the understanding of this study. In a lab experiment, the sample is excited at a frequency corresponding to a loworder compressional resonance mode (typically a few $\mathrm{kHz}$ ). The dynamic change of elasticity induced by this stationary elastic wave is measured by applying simultaneously a sequence of ultrasonic short bursts (with a typical center frequency of $1 \mathrm{MHz}){ }^{31}$ The geometry of the sample and the position of the ultrasound transducers that transmit and receive the ultrasonic pulses are selected so that the strain field (produced by the stationary elastic wave) traversed by the ultrasonic pulses is quasi-homogeneous and quasi-static with regard to the ultrasonic travel time in the sample. Each ultrasonic pulse experiences a different strain level, and the large number of ultrasonic pulses in the sequence (1000) provides a dense sampling of the relation between the ultrasonic wave-speed and applied strain. The change of wavespeed is deduced from the change of travel time. The latter is precisely determined with a method based on the crosscorrelation function between a given ultrasonic pulse of the sequence and the very first ultrasonic pulse that serves as a reference (for more details, see for instance Ref. 31). The strain value associated with each ultrasonic pulse is a spatial and temporal average of the actual strain seen by the ultrasonic pulse during its propagation through the sample. ${ }^{30}$ Contrary to conventional quasi-static measurements of the acousto-elastic effect, ${ }^{16}$ we do not glue the ultrasonic transducers on the sample, the transducers are placed a few millimeters away from the sample, and coupling is ensured with an ultrasound transmission gel. The changes in the ultrasonic path length in the coupling gel due to the deformation of the sample (while ultrasound transducers are immobile) must be corrected. ${ }^{30}$ For porous materials like Berea sandstone, a thin layer of nail polish is applied to the contact area so that the gel does not penetrate by capillary action. In this work, a shear ultrasonic wave with vertical polarisation is generated and received without shear ultrasound transducers and without direct contact with the sample. It relies on the refraction of a compressional ultrasonic wave generated by a compressional ultrasound transducer (Figure 1). Details on the signal processing and the choice of the incident angle are addressed in Appendix A.

\section{B. Relation between the change of ultrasonic wave-speed induced by a uniaxial loading and third-order elastic constants (TOECs) for an initially isotropic medium}

Under the approximation of a third-order hyperelastic model (based on the assumption that the strain energy density function is a third-order polynomial), there are three independent TOECs for an isotropic elastic material. We use here the Murnaghan third-order elastic constants $l, m$, and $n$. The equations in the case of a uniaxial loading along the y-dimension (Figure 1) and the arbitrary angle between the loading direction and the direction of ultrasonic propagation are recalled, for the velocity of a compressional wave (P-wave) $V_{P}$ and a shear wave with polarisation in the $(x, y)$ plane (SV-wave) $V_{S V}{ }^{32}$

$$
\begin{aligned}
\rho_{0} V_{P}^{2}= & (\lambda+2 \mu)+\frac{\epsilon_{y y}}{(\lambda+\mu)}\left[\left(\sin \theta_{P}\right)^{2}\left(2 \lambda^{2}+9 \lambda \mu+6 \mu^{2}\right)\right. \\
& -\left(\cos \theta_{P}\right)^{2} \lambda(\lambda+2 \mu)+2 \mu l \\
& \left.+\left(4\left(\sin \theta_{P}\right)^{2}(\lambda+\mu)-2\left(\cos \theta_{P}\right)^{2} \lambda\right) m\right],
\end{aligned}
$$


(a)

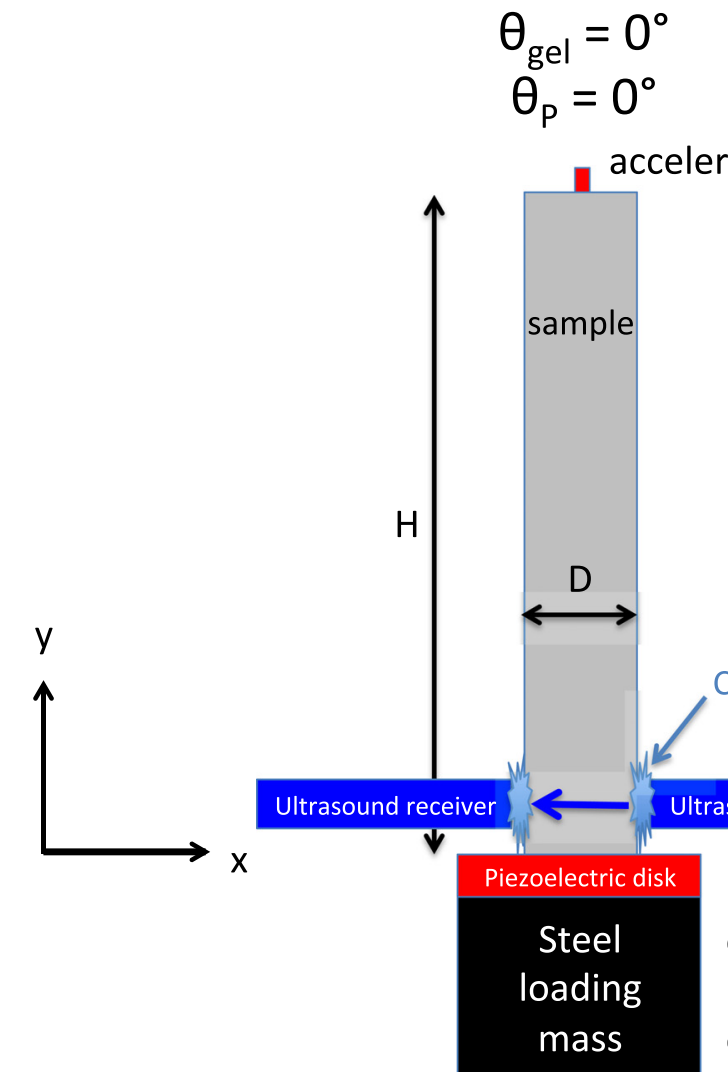$$
\theta_{p}=0^{\circ}
$$

accelerometer

FIG. 1. Experimental setup. (a) Experimental configuration for the first type of measurement with normal incidence. (b) Experimental configuration for the second and third types of measurements with oblique incidence. $h_{\text {probe }}$ is the vertical distance between the clamped end of the sample and the entering point of the ultrasonic beam in the sample.

$$
\rho_{0} V_{S V}^{2}=\mu+\frac{\epsilon_{y y}}{(\lambda+\mu)}\left[2 \mu(\lambda+\mu)+\mu m+\frac{\lambda}{4} n\right] .
$$

$V_{P}$ and $V_{S V}$ are natural velocities, i.e., the propagation distance in the undeformed state divided by the propagation time. ${ }^{10}$ The subscript 0 means at zero applied strain. $\rho_{0}$ is the mass density. $\lambda$ and $\mu$ are Lamé second order elastic constants. $\theta_{P}$ is the refraction angle for the compressional wave in the sample determined by Snell's law; $\sin \left(\theta_{P}\right)=\sin \left(\theta_{\text {gel }}\right) V_{P_{0}} / V_{\text {gel }}$, where $\theta_{\text {gel }}$ and $V_{\text {gel }}$ are the incidence angle and the compressional wavespeed in the coupling gel. $\epsilon_{y y}$ is the strain experienced by an ultrasonic pulse during its propagation in the sample. The derivation of these equations assumes that the applied stress is uniform and static. ${ }^{10}$ We apply these equations to DAET since the applied stress is quasi-static and quasi-uniform.

We shall later in this manuscript measure the first-order derivative of the relative variation of wave-speeds $V_{P}$ and $V_{S V}$, i.e., $\frac{\Delta V_{P}}{V_{P_{0}}}=\frac{V_{P}-V_{P_{0}}}{V_{P_{0}}}$ and $\frac{\Delta V_{S V}}{V_{S V_{0}}}=\frac{V_{S V}-V_{S V_{0}}}{V_{S V_{0}}}$. From Equations (1) and (2), one obtains

$$
\begin{aligned}
\frac{\partial}{\partial \epsilon_{y y}}\left(\frac{\Delta V_{P}}{V_{P_{0}}}\right)= & \frac{1}{2(\lambda+2 \mu)(\lambda+\mu)}\left[\left(\sin \theta_{P}\right)^{2}\left(2 \lambda^{2}+9 \lambda \mu+6 \mu^{2}\right)\right. \\
& -\left(\cos \theta_{P}\right)^{2} \lambda(\lambda+2 \mu)+2 \mu l \\
& \left.+\left(4\left(\sin \theta_{P}\right)^{2}(\lambda+\mu)-2\left(\cos \theta_{P}\right)^{2} \lambda\right) m\right],
\end{aligned}
$$

(b)

$$
\begin{gathered}
\theta_{\text {gel }}=25^{\circ} \\
\theta_{P}=54^{\circ}(\text { Berea }), 45^{\circ}(\text { PMMA }) \\
\theta_{S V}=28^{\circ} \text { (Berea), } 21^{\circ}(\text { PMMA })
\end{gathered}
$$

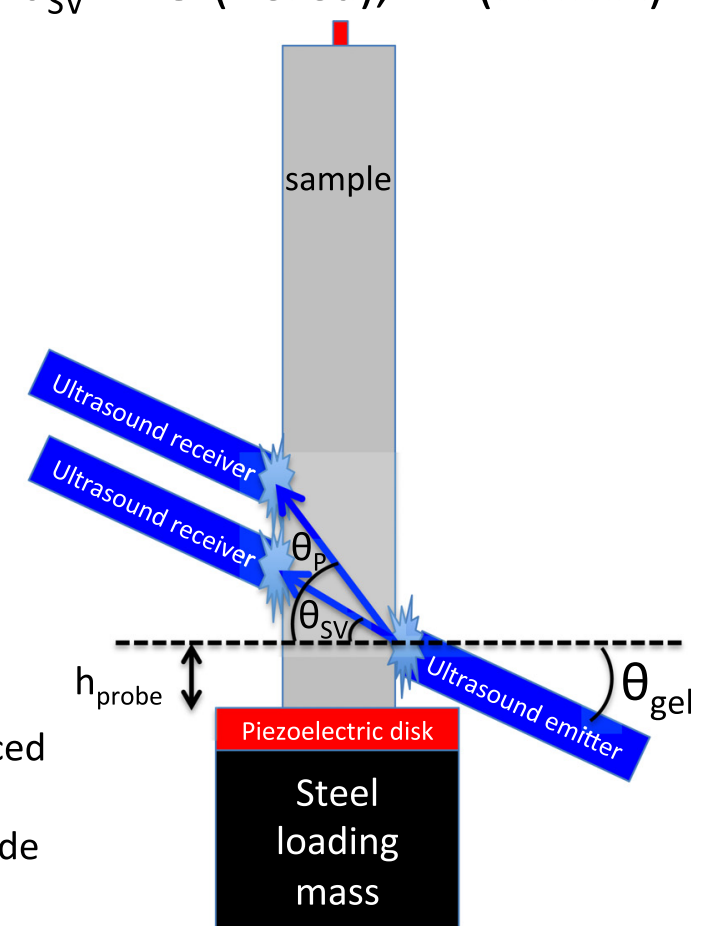


TABLE I. Measured geometry and physical properties of the samples.

\begin{tabular}{lcc}
\hline \hline Material & PMMA & Berea sandstone \\
\hline Diameter $(\mathrm{mm})$ & 30 & 25 \\
Length $(\mathrm{mm})$ & 200 & 150 \\
Mass density $\rho_{0}\left(\mathrm{~kg} / \mathrm{m}^{3}\right)$ & $1172 \pm 1 \%$ & $2152 \pm 1 \%$ \\
Permeability $(\mathrm{mD})$ & & $100-200$ \\
Bulk compressional wave-speed $V_{P_{0}}(\mathrm{~m} / \mathrm{s})$ & $2730 \pm 1 \%$ & $3090 \pm 2.5 \%$ \\
Bulk shear wave-speed $V_{S_{0}}(\mathrm{~m} / \mathrm{s})$ & $1385 \pm 1 \%$ & $1810 \pm 2.5 \%$ \\
Frequency of resonance mode $(\mathrm{Hz})$ & 2794 & 4610 \\
\hline \hline
\end{tabular}

sandstone (Cleveland quarry near Birmingham, OH, USA, gray sandstone quarried approximately $20 \mathrm{ft}$ from the bottom of the quarry, in the lower formation of the west side of the quarry). During the experiments, the room temperature was close to $20^{\circ} \mathrm{C}$ and the relative humidity varied between $50 \%$ and $80 \%$. Prior to DAET experiments, bulk compressional and shear wave-speeds were measured with a dedicated setup. The characteristics of the samples are summarized in Table I. We measured the bulk compressional wave-speed in the transmission coupling gel $V_{\text {gel }}=1620 \mathrm{~m} / \mathrm{s} \pm 2.5 \%$. The second order elastic constants $\lambda$ and $\mu$ were calculated from the measurements of the mass density $\rho_{0}$, the bulk compressional wave-speed $V_{P_{0}}$, and the bulk shear wave-speed $V_{S_{0}}$ as $\mu=\rho_{0} V_{S_{0}}^{2}$ and $\lambda=\rho_{0}\left(V_{P_{0}}^{2}-2 V_{S_{0}}^{2}\right)$.

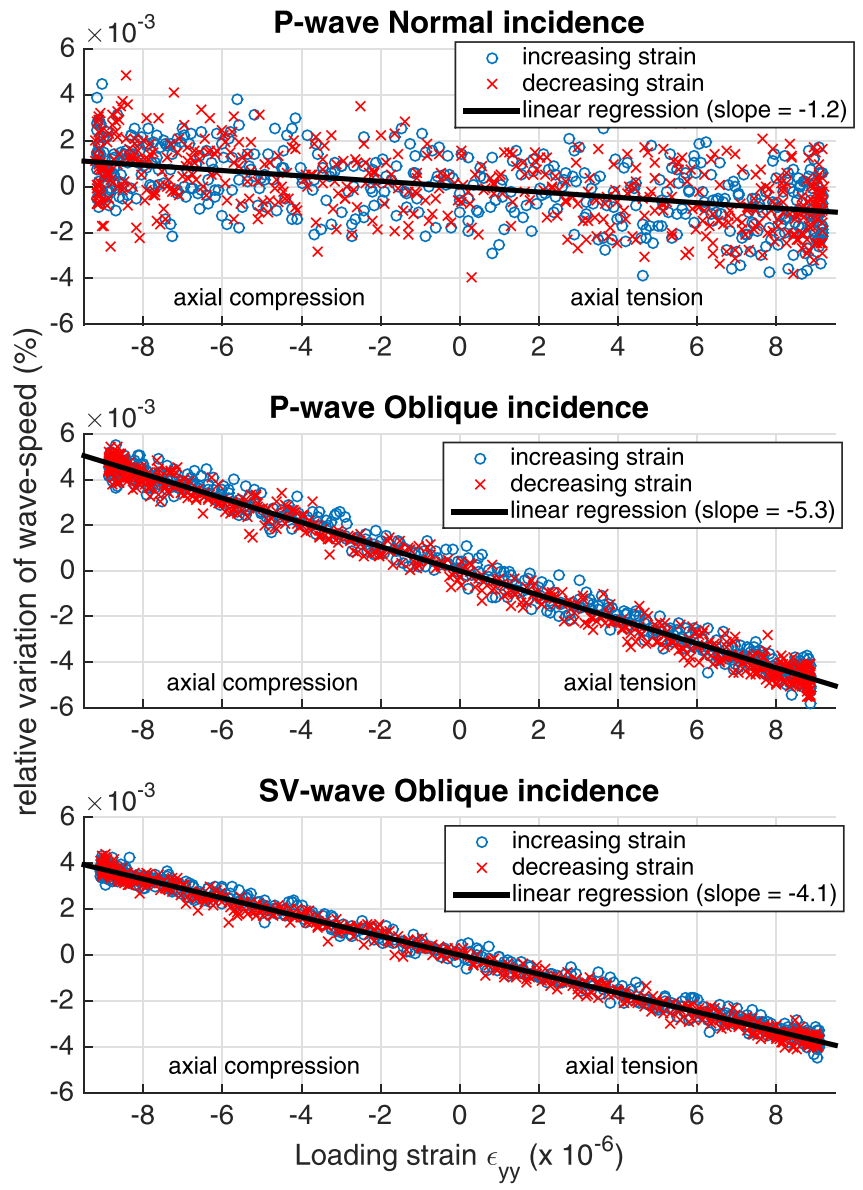

FIG. 2. Typical measurements in PMMA: relative change of ultrasonic wave-speed as a function of the axial loading strain $\left(\epsilon_{y y}\right)$. Blue circles indicate decreasing strain while red crosses indicate increasing strain. The black solid lines show the result of the linear regression.
In order to quantify the impact of the variability due to the positioning of the ultrasound transducers on the estimation of the TOECs, the measurements were repeated multiple times with systematic removal and repositioning of the two ultrasound transducers. Each type of measurement was repeated 12 times for Berea sandstone and 9 times for PMMA, during 3 different days. By doing so, we aimed to measure the TOECs with evaluation of the uncertainty of the estimates, including the effect of the reproducibility of the measurements. Figures 2 and 3 show typical measurements in PMMA and Berea sandstone, respectively. The theory (Equations (3) and (4)) predicts a linear relation between the relative change of wave-speed and the applied strain. Therefore, the first-order derivative of the relation between the relative change of wave-speed $\left(\frac{\partial}{\partial \epsilon_{y y}}\left(\Delta V_{P} / V_{P_{0}}\right)\right.$ or $\left.\frac{\partial}{\partial \epsilon_{y y}}\left(\Delta V_{S V} / V_{S V_{0}}\right)\right)$ and the axial strain $\left(\epsilon_{y y}\right)$ was determined by applying a linear regression to the 1000 data points. Each data point was obtained from the analysis of a single ultrasonic pulse that experienced a given strain level. In PMMA, a linear function fitted the experimental data very well (Figure 2). In Berea sandstone, the relation between the relative change of wave-speed and the loading strain $\epsilon_{y y}$ was more complex (Figure 3), as hysteresis and a DC offset were observed. Previous work ${ }^{26,27,31,33}$ showed that the hysteresis and the DC offset observed in rocks depend on the strain amplitude and frequency of the dynamic loading. However, the first-order derivative of the relation between the relative

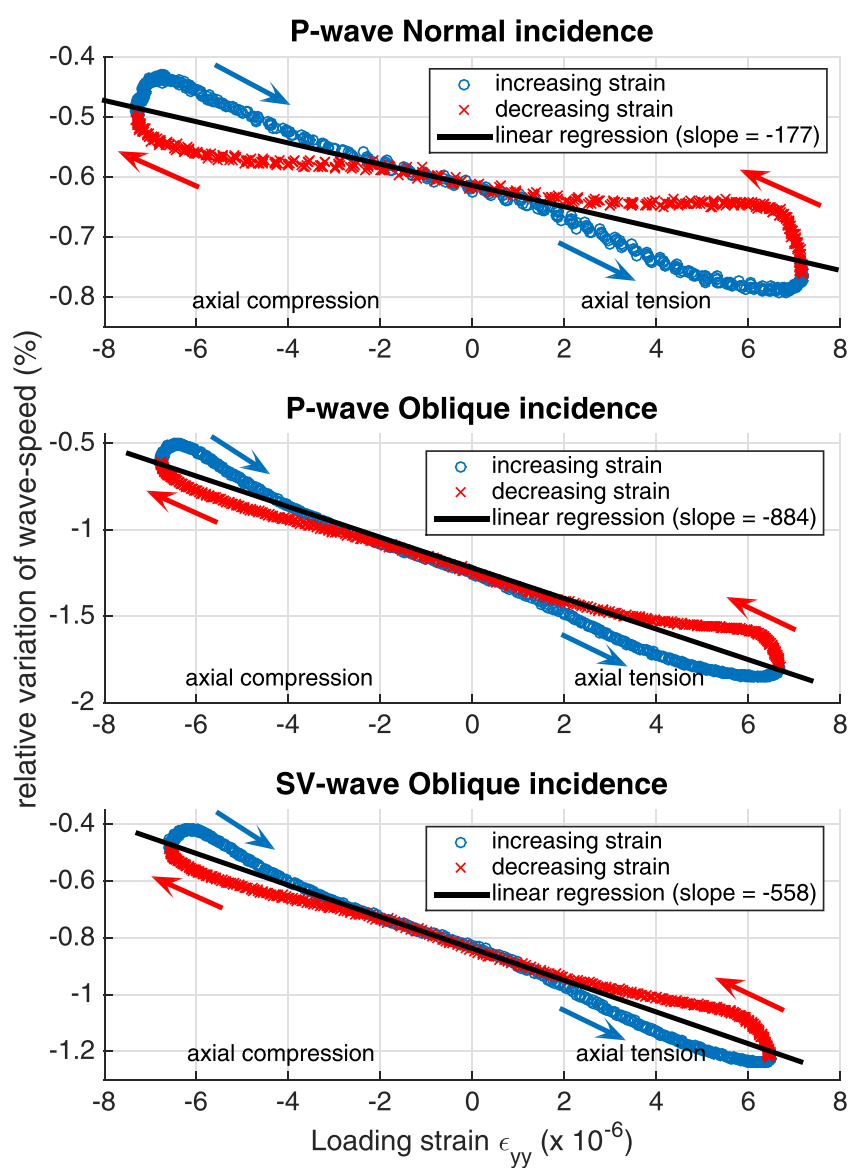

FIG. 3. Typical measurements in Berea sandstone: relative change of ultrasonic wave-speed as a function of the axial loading strain $\left(\epsilon_{y y}\right)$. Blue circles indicate decreasing strain while red crosses indicate increasing strain. The black solid lines show the result of the linear regression. 
change of the wave-speed and the loading strain (i.e., the mean slope in Figures 2 and 3) shows virtually no sensitivity to the strain amplitude and frequency. Therefore, we propose to model this feature of the nonlinear elastic behavior of Berea sandstone by applying the classical theory of nonlinear elasticity involving the TOECs.

\section{Estimation of the third-order elastic constants and uncertainty}

Measurements were repeated 12 times for Berea sandstone and 9 times for PMMA; therefore, the TOECs are estimated by applying overdetermined linear regression (see for instance Refs. 34 and 35). The relation between the observations, the model, and the three unknown parameters can be written in the form of a matrix product $y=A x+d+e$, where $A$ is a $3 N \times 3$ matrix, and $y, x, d$, and $e$ are $3 N$-dimensional vectors. $N$ is the number of repetition of the measurement, $N=9$ for PMMA and $N=12$ for Berea sandstone. $y$ contains the observations while $x$ is a column vector containing the three unknown parameters (i.e., the third-order elastic constants). The vector $e$ contains the errors on the measured values $y . A$ and $d$ are constructed with coefficients that are determined by the model, and they link the observations to the three unknown TOECs (Equations (3) and (4))

$$
\left(\begin{array}{c}
\left.\frac{\partial}{\partial \epsilon_{y y}}\left(\frac{\Delta V_{P}}{V_{P_{0}}}\right)\right|_{i=1} ^{\theta_{\mathrm{gel}}=0^{\circ}} \\
\vdots \\
\left.\frac{\partial}{\partial \epsilon_{y y}}\left(\frac{\Delta V_{P}}{V_{P_{0}}}\right)\right|_{i=N} ^{\theta_{\mathrm{gel}}=0^{\circ}} \\
\left.\frac{\partial}{\partial \epsilon_{y y}}\left(\frac{\Delta V_{P}}{V_{P_{0}}}\right)\right|_{i=1} ^{\theta_{\mathrm{gel}}=25^{\circ}} \\
\left.\frac{\partial}{\partial \epsilon_{y y}}\left(\frac{\Delta V_{P}}{V_{P_{0}}}\right)\right|_{i=N} ^{\theta_{\mathrm{gel}}=25^{\circ}} \\
\left.\frac{\partial}{\partial \epsilon_{y y}}\left(\frac{\Delta V_{S V}}{V_{S V_{0}}}\right)\right|_{i=1} ^{\theta_{\mathrm{gel}}=25^{\circ}} \\
\left.\frac{\partial}{\partial \epsilon_{y y}}\left(\frac{\Delta V_{S V}}{V_{S V_{0}}}\right)\right|_{i=N} ^{\theta_{\mathrm{gel}}=25^{\circ}}
\end{array}\right)=\left(\begin{array}{ccc}
a_{1} & b_{1} & 0 \\
\vdots & \\
a_{1} & b_{1} & 0 \\
a_{2} & b_{2} & 0 \\
\vdots & \\
a_{2} & b_{2} & 0 \\
0 & b_{3} & c_{3} \\
\vdots \\
0 & b_{3} & c_{3}
\end{array}\right)\left(\begin{array}{c}
l \\
m \\
d_{1} \\
d_{2} \\
\vdots \\
d_{2} \\
d_{3} \\
\vdots \\
d_{3}
\end{array}\right)+\left(\begin{array}{c}
e_{1} \\
\vdots \\
e_{3 N}
\end{array}\right)+
$$

The coefficients $a_{1}, b_{1}, d_{1}, a_{2}, b_{2}, d_{2}, b_{3}, c_{3}$, and $d_{3}$ are given by

$$
\begin{aligned}
& a_{1}=\frac{\mu}{(\lambda+2 \mu)(\lambda+\mu)}, \\
& b_{1}=-\frac{\lambda}{(\lambda+2 \mu)(\lambda+\mu)}, \\
& d_{1}=-\frac{\lambda}{2(\lambda+\mu)}, \\
& a_{2}=\frac{\mu}{(\lambda+2 \mu)(\lambda+\mu)} \\
& b_{2}=\frac{2\left(\sin \theta_{P}\right)^{2}(\lambda+\mu)-\left(\cos \theta_{P}\right)^{2} \lambda}{(\lambda+2 \mu)(\lambda+\mu)}, \\
& d_{2}=\frac{\left(\sin \theta_{P}\right)^{2}\left(2 \lambda^{2}+9 \lambda \mu+6 \mu^{2}\right)-\left(\cos \theta_{P}\right)^{2} \lambda(\lambda+2 \mu)}{2(\lambda+2 \mu)(\lambda+\mu)} \\
& b_{3}=\frac{1}{2(\lambda+\mu)}, \\
& c_{3}=\frac{\lambda}{8 \mu(\lambda+\mu)}, \\
& d_{3}=1
\end{aligned}
$$

The least-square estimate of the three unknown TOECs $\hat{x}$ is given by

$$
\hat{x}=\left(A^{T} A\right)^{-1} A^{T}(y-d),
$$

and the corresponding covariance matrix of the estimates of the TOECs by

$$
\operatorname{Var}(\hat{x})=\hat{\sigma}^{2}\left(A^{T} A\right)^{-1}
$$

where $\hat{\sigma}^{2}$ is the mean squared error of the fitted model. The square roots of the diagonal elements of this matrix are the standard deviations of the estimates of the TOECs.

The uncertainty of the estimation of the TOECs was evaluated with Monte-Carlo simulations (200000 samples). For each sample, we repeated the following steps. For each parameter, random values were generated following a normal distribution with a mean value equal to the nominal value of the parameter and a standard deviation equal to the uncertainty given in Table II. Table II lists the parameters and their uncertainty involved in the inference of the 
TABLE II. Uncertainty of the parameters involved in the inference of the TOECs.

\begin{tabular}{lc}
\hline \hline Parameter & Uncertainty \\
\hline Compressional wave-speed (PMMA) & $\pm 1 \%$ \\
Shear wave-speed (PMMA) & $\pm 1 \%$ \\
Compressional wave-speed (Berea) & $\pm 2.5 \%$ \\
Shear wave-speed (Berea) & $\pm 2.5 \%$ \\
Compressional wave-speed (coupling gel) & $\pm 2.5 \%$ \\
Mass density & $\pm 1 \%$ \\
Accelerometer sensitivity & $\pm 1 \%$ \\
Incidence angle in coupling gel & $\pm 1^{\circ}$ \\
\hline \hline
\end{tabular}

TOECs. The coefficients in $A$ and $d$ were computed. Linear regression was applied to obtain an estimate of the TOECs.

Figure 4 shows the results of Monte-Carlo simulations for the TOECs. The median values of the distributions of the TOECs are $l=-73 \mathrm{GPa}, m=-34 \mathrm{GPa}$, and $n=-61 \mathrm{GPa}$ for PMMA. For Berea sandstone, we find $l=-17000 \mathrm{GPa}$, $m=-11000 \mathrm{GPa}$, and $n=-30000 \mathrm{GPa}$. For PMMA, the lower limit of the second quartile and the upper limit of the
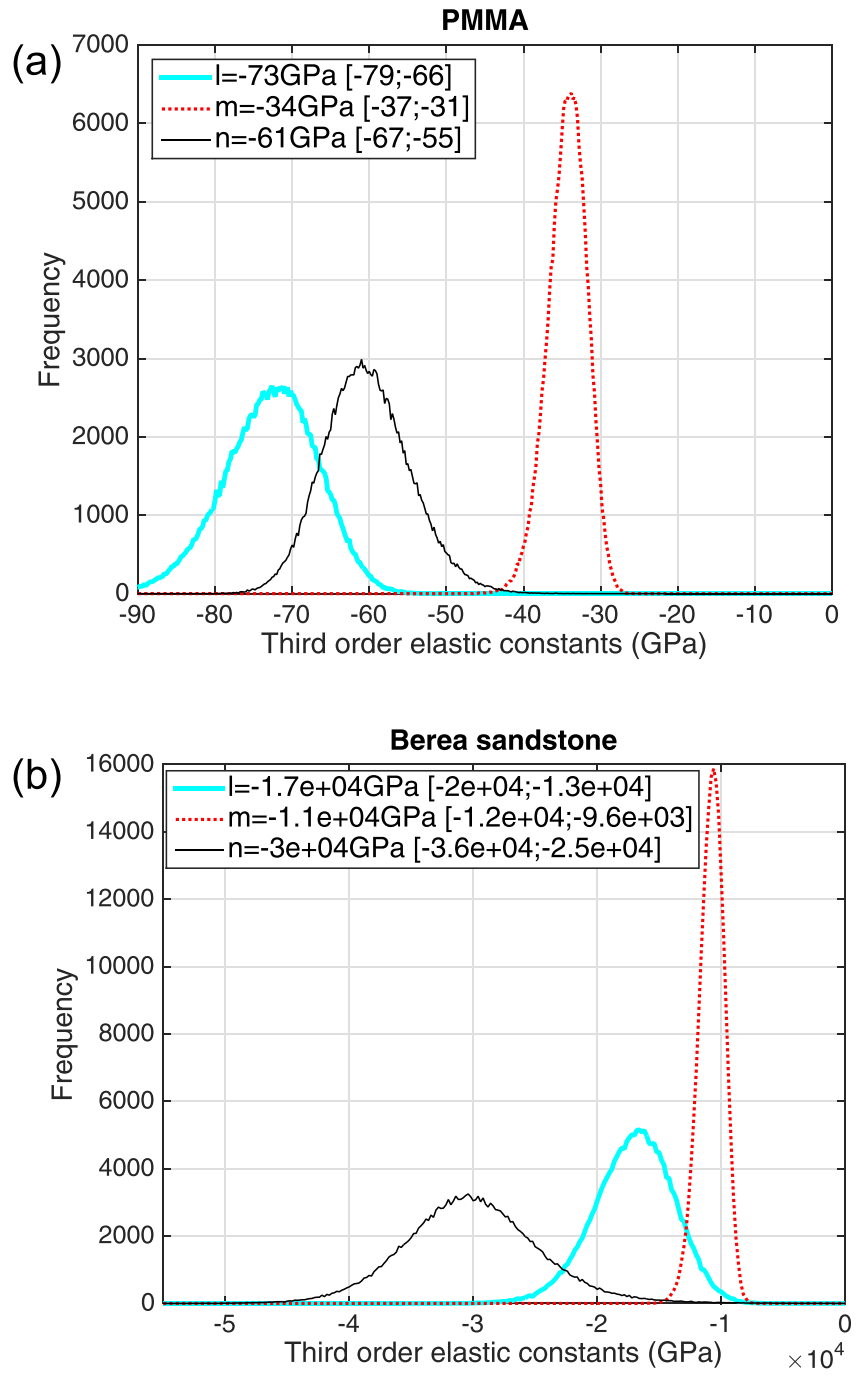

FIG. 4. Distribution of the TOECs $l, m$, and $n$ obtained with Monte-Carlo simulations for PMMA (top) and Berea sandstone (bottom). The median value and the inter-quartile range are indicated. third quartile are found at $-9 \%$ and $+9 \%$ about the median values for $l$ and $m$, and $-10 \%$ and $+10 \%$ for $n$. For Berea sandstone, the lower limit of the second quartile and the upper limit of the third quartile are found at $-20 \%$ and $+20 \%$ about the median values of $l,-10 \%$ and $+10 \%$ about the median values of $m$, and $-20 \%$ and $+20 \%$ about the median values of $n$. Uncertainty (in percent) is larger for Berea sandstone than for PMMA; this is likely essentially due to the slight inhomogeneity of the sample of Berea sandstone and the dependence on temperature and humidity conditions of its elastic properties. This is supported by the fact that the reproducibility of the measurement in Berea sandstone is worse than in PMMA (Table III).

\section{DISCUSSION}

\section{A. Comparison of TOECs measured in this work in PMMA and Berea sandstone with literature}

In this first part of the Discussion, we propose to compare the values of the TOECs obtained in this work applying DAET to studies that reported either measurements of all three TOECs in PMMA and Berea sandstone or measurements giving access to a single coefficient of elastic nonlinearity that is a combination of the TOECs and the second-order elastic constants. Although this comparison is necessary, it is worth mentioning that its relevance is limited by the variability of the elastic properties of the materials. While the variability of the elastic properties of rock samples extracted at different places in a quarry is obvious, the variability of the elastic properties for PMMA was also mentioned in the literature. ${ }^{36}$

\section{Comparison with conventional quasi-static acousto-elastic measurements}

Table IV shows the TOECs obtained in this study and by Winkler et al. ${ }^{16}$ in PMMA and Berea sandstone. The standard deviations of the estimates obtained in this work are given

TABLE III. Mean and standard deviation (SD) of the linear coefficient relating the relative change of wave-speed to the strain measured in PMMA $(N=9$ repetitions of the measurement) and Berea sandstone $(N=12$ repetitions of the measurement). Standard deviations are given in absolute values and as a percent of the mean value.

\begin{tabular}{cccc}
\hline \hline & Incidence & $\begin{array}{c}\text { PMMA } \\
\text { Mean }(N=9)\end{array}$ & $\begin{array}{c}\text { PMMA } \\
\text { SD }(N=9)\end{array}$ \\
\hline$\frac{\partial}{\partial \epsilon_{y y}}\left(\left[\Delta V_{P}\right] / V_{P_{0}}\right)$ & Normal & -1.2 & $0.085(7 \%)$ \\
$\frac{\partial}{\partial \epsilon_{y y}}\left(\left[\Delta V_{P}\right] / V_{P_{0}}\right)$ & Oblique & -5.4 & $0.11(2 \%)$ \\
$\frac{\partial}{\partial \epsilon_{y y}}\left(\left[\Delta V_{S V}\right] / V_{S V_{0}}\right)$ & Oblique & -4.1 & $0.31(8 \%)$ \\
& & Berea sandstone & Berea sandstone \\
$\frac{\partial}{\partial \epsilon_{y y}}\left(\left[\Delta V_{P}\right] / V_{P_{0}}\right)$ & Normal & -171 & $16(9 \%)$ \\
$\frac{\partial}{\partial \epsilon_{y y}}\left(\left[\Delta V_{P}\right] / V_{P_{0}}\right)$ & Oblique & -970 & $110(11 \%)$ \\
$\frac{\partial}{\partial \epsilon_{y y}}\left(\left[\Delta V_{S V}\right] / V_{S V_{0}}\right)$ & Oblique & -631 & $140(22 \%)$ \\
\hline \hline
\end{tabular}


TABLE IV. Estimates of the TOECs and standard deviations of the estimates obtained in this study and reported by Winkler et al. ${ }^{16}$ in PMMA and dry Berea sandstone. Two samples of Berea sandstone (Berea 1 and Berea 2) were studied in Ref. 16.

\begin{tabular}{lccr}
\hline \hline & $l(\mathrm{GPa})$ & $m(\mathrm{GPa})$ & \multicolumn{1}{c}{$n(\mathrm{GPa})$} \\
\hline PMMA (this study) & $-73 \pm 2 \%$ & $-34 \pm 2 \%$ & $-61 \pm 4 \%$ \\
PMMA $^{16}$ & $-37 \pm 16 \%$ & $-29 \pm 21 \%$ & $-22 \pm 27 \%$ \\
Berea (this study) $^{17000 \pm 7 \%}$ & $-11000 \pm 7 \%$ & $-30000 \pm 19 \%$ \\
Berea 1 $^{16}$ & $-1850 \pm 2 \%$ & $-4200 \pm 2 \%$ & $-5010 \pm 3 \%$ \\
Berea 2 $^{16}$ & $-2040 \pm 26 \%$ & $-4260 \pm 10 \%$ & $-5640 \pm 6 \%$ \\
\hline \hline
\end{tabular}

and can be compared to those reported in Ref. 16; it evaluates the goodness of fit between the measurements and the model (the uncertainty of estimates was not provided by the authors $\left.{ }^{16}\right)$. We obtained TOECs in Berea sandstone that are larger than those reported in Ref. 16. As noted by the authors in Ref. 16, their conventional quasi-static acousto-elastic measurements were conducted at a strain level that exceeds the domain of validity of the model (see Section III B 2 for further details). Consequently, the authors proposed to extrapolate at zero stress the stress-dependence of the ultrasonic wave-speed measured at few MPa applied stress. This procedure was also applied by Johnson et al. ${ }^{37}$ Therefore, we believe that the measurements conducted by Winkler et al. ${ }^{16}$ may have led to approximate values of TOECs. In contrast, DAET operates at microstrains about the equilibrium state (zero stress), within the domain of validity of the third-order hyperelastic model. Beyond this direct comparison for Berea sandstone, it is worth mentioning that other authors have reported the values of TOECs in a different type of sandstone close to $-100000 \mathrm{GPa}$ applying conventional quasi-static acousto-elastic measurements, ${ }^{37}$ i.e., larger than the values reported in this work in Berea sandstone.

For PMMA, we obtain TOECs that are significantly larger than those reported by Winkler et al. ${ }^{16}$ However, the authors pointed out that the variation of ultrasonic wavespeed with stress was small and rather difficult to measure with their techniques. In this work, we use a method based on the cross-correlation function refined with parabolic interpolation of the maximum of the cross-correlation function (for more details, see for instance Ref. 31). This is a very robust technique to detect a subsample time shift. As seen in Table IV, the standard deviations of the estimates that evaluate the goodness of fit between the measurements and the model are smaller in this work than in the work by Winkler et al. ${ }^{16}$

From the TOECs measured in PMMA applying DAET, we can derive a coefficient that quantifies the dependence of the compressional wave-speed $V_{P}$ on the hydrostatic pressure $P$. In this way, our results can be compared to other studies reported in the literature. The dependence of the compressional wave-speed $V_{P}$ on the hydrostatic pressure $P$ is determined by the TOECs and the second-order elastic constants: $\rho_{0} \partial V_{P}^{2} / \partial P=-(6 l+4 m+7 \lambda+10 \mu) /(3 \lambda+2 \mu) . \quad$ Asay et $a l .{ }^{15}$ measured $\rho_{0} \partial V_{P}^{2} / \partial P=16$ and Renaud et al. ${ }^{38}$ reported $\rho_{0} \partial V_{P}^{2} / \partial P=12.4$. We find $\rho_{0} \partial V_{P}^{2} / \partial P=30 \pm 5 \%$ (the uncertainty is half the interquartile range obtained with
Monte-Carlo simulations), therefore larger than the two values reported in the literature.

It is important to highlight that the uncertainty of the estimation of the TOECs (Figure 4) is larger than the standard deviations of the fitting process (Table IV), because it includes the effects of reproducibility and uncertainty of the other parameters (Table II). The reproducibility of conventional quasi-static acousto-elastic measurements was rarely reported in the literature, ${ }^{39}$ while it is a good practice to report the estimates of physical constants with their overall uncertainty. It is also worth stressing that the accuracy of the estimation of the TOECs is highly dependent on the accuracy of the estimation of the parameters, particularly the velocity of compressional and shear waves and the incidence angle in the coupling gel. Our experimental setup can definitely be further refined to improve the reproducibility of the measurement and therefore reduce the associated variability of the measurement (Table III). In particular, the system used to maintain and position the ultrasound transducers with a certain incidence angle was not optimal. Thus, we speculate that TOECs in a homogeneous material like PMMA can be estimated with DAET with an uncertainty of less than 5\%, with an optimal experimental setup.

\section{Comparison with harmonic generation measurements}

The nonlinear propagation of a compressional elastic wave is often described with the parameter of nonlinearity $\beta$. A typical experiment consists in broadcasting a monofrequency ultrasonic burst and recording the signal generated by nonlinear propagation at the second harmonic frequency (twice the frequency injected in the medium). The parameter of nonlinearity $\beta$ is derived from the amplitude of the component at the second harmonic frequency and it is related to the TOECs by

$$
\beta=-\left(\frac{3}{2}+\frac{l+2 m}{\lambda+2 \mu}\right) .
$$

Using the values of the third-order and second-order elastic constants obtained in this study for Berea sandstone, we obtain $\beta=1900 \pm 6 \%$ (the uncertainty is half the interquartile range obtained with Monte-Carlo simulations). In this way, our results can be compared to the ultrasonic harmonic generation measurements reported in the literature. The comparison of the TOECs obtained applying DAET to harmonic generation measurements is particularly interesting because the two techniques are operated in the same range of strain (of the order of $10^{-6}$ ). Applying ultrasonic harmonic generation measurements in Berea sandstone, Meegan et al. ${ }^{40}$ found $\beta=7000 \pm 25 \%$, and Tencate et $_{\text {al }}{ }^{41}$ reported $\beta=400$.

For PMMA, Landsberger et al. ${ }^{42}$ obtained $\beta=10$ applying ultrasonic harmonic generation measurements. We find $\beta=14.6 \pm 5 \%$ (the uncertainty is half the interquartile range obtained with Monte-Carlo simulations), therefore in good agreement with the work by Landsberger et al. ${ }^{42}$ 


\section{B. Accuracy of the acousto-elastic measurement of TOECs}

Our goal here is to show that the accuracy of the acousto-elastic measurement of the TOECs can be improved in several ways. In particular, the actual applied stress experienced by ultrasonic bursts must be precisely evaluated, the signal processing technique for measuring changes of ultrasonic travel time must be optimal, as small applied stress as possible must be applied, and the differences between adiabatic and isothermal second-order elastic constants and their frequency-dispersion must be considered.

\section{Accurate calculation of applied stress along ultrasonic path}

Quasi-static acousto-elastic measurements applying a uniaxial compressive loading often assumes perfect sliding conditions at the interface between the sample and the plates of the testing machine, as well as perfect parallelism. ${ }^{43}$ Under these conditions, the stress field in the sample is uniform and the actual stress in the sample is correctly evaluated. However, such ideal conditions are never met and partial adhesion occurs. Although it was proposed to minimize lateral constraint by using indium, lead, or teflon shims, ${ }^{12,44}$ this issue has received little attention. Johnson et al. ${ }^{45}$ showed that, for a rectangular parallelepipedic sample, a slenderness ratio of at least 8 is necessary to measure properly the stress dependence of a compressional wave when the ultrasonic transducers are placed in the central region of the sample. They found that the use of a sample with a small slenderness ratio leads to the underestimation of the actual stress-dependence of the ultrasonic wave-speed. In particular, the use of a cubical sample underestimates by $24 \%$ the actual stress-dependence of the ultrasonic wave-speed.

Winkler et al. ${ }^{16}$ conducted conventional quasi-static acousto-elastic measurements in PMMA and Berea sandstone. They used prism-shaped samples of $2.5 \mathrm{~cm} \times 7.5 \mathrm{~cm} \times 15 \mathrm{~cm}$, that is to say, with a slenderness ratio of only 2 in one plane. The ultrasound transducers are placed at mid-length of the sample, and the actual stress experienced by an ultrasonic burst is assumed to be given by the compressive force applied by the testing machine and the section area of the sample $(2.5 \mathrm{~cm} \times 7.5 \mathrm{~cm})$. Therefore, the stress field was assumed uniform. As a consequence, the actual static stress along the ultrasonic path may have been overestimated since such a configuration leads to a non-uniform stress field. The two ends of the sample in contact with the plates of the testing machine experience a higher stress than the center of the sample that is traversed by the ultrasonic pulses. In DAET, the stress field is not uniform but can be calculated; ${ }^{46}$ it is determined by the first axial compressional resonance mode of the cylindrical sample (see Appendix B for more details on the correction of the non-uniformity of the applied strain field).

\section{Validity of theory of elasticity}

Experiments of acousto-elasticity are classically operated with the assumption that the elastic strain energy $W$ can be approximated by a Taylor expansion in strain up to the thirdorder. For an isotropic solid, the elastic strain energy reduces to ${ }^{3}$

$$
\begin{aligned}
\rho_{0} W= & \frac{\lambda}{2}(\operatorname{tr} \epsilon)^{2}+\mu \operatorname{tr} \epsilon^{2}+\frac{2 l-2 m+n}{6}(\operatorname{tr} \epsilon)^{3} \\
& +\frac{2 m-n}{2}(\operatorname{tr} \boldsymbol{\epsilon}) \operatorname{tr} \epsilon^{2}+\frac{n}{3} \operatorname{tr} \epsilon^{3},
\end{aligned}
$$

where $\operatorname{tr} \epsilon$ is the trace of the strain tensor $\epsilon$ and $\rho_{0}$ is the unstrained mass density. This development of the elastic strain energy is valid as long as the three terms involving the TOECs $(l, m$, and $n)$ remain much smaller than the first two terms involving the second-order elastic constants $(\lambda$ and $\mu$ ). Using the values of the third-order and second-order elastic constants obtained in this study and taking $\epsilon_{y y}=10^{-4}$, it is found that the energy attributed to nonlinear elastic strain is $10 \%$ of the energy attributed to linear elastic strain.

Winkler et al. ${ }^{16}$ applied a strain larger than $10^{-4}$ (applied stress was varied from $0.5 \mathrm{MPa}$ to $6 \mathrm{MPa}$ ), and the authors write "It should be noted that third-order elasticity predicts linear plots of velocity-squared versus stress, so the fact that we observe curvature in some rocks shows that the assumptions of the theory have been exceeded." In contrast, the applied strain in DAET is much smaller. With $\epsilon_{y y}=8$ $\times 10^{-6}$, the energy attributed to nonlinear elastic strain is less than $1 \%$ of the energy attributed to linear elastic strain. We obtained the values of TOECs in Berea sandstone that are significantly larger than those reported by Winkler et al. ${ }^{16}$ This discrepancy may be due to the fact that their measurements were conducted outside the range of validity of the theory of third-order elasticity.

\section{Adiabatic and isothermal second-order elastic constants-Frequency dispersion of second-order elastic constants}

The theory developed for conventional quasi-static experiments of acousto-elasticity ${ }^{10}$ involves isothermal elastic constants and adiabatic elastic constants. The applied strain is generated in a quasi-static manner; therefore, the process is isothermal, while ultrasound propagation is an adiabatic phenomenon. Although small, the difference between isothermal elastic constants and adiabatic elastic constants is virtually systematically neglected. ${ }^{34,37}$ In contrast, DAET involves solely the adiabatic elastic constants since the applied strain is dynamic with a frequency of a few $\mathrm{kHz}$.

In addition, in materials like PMMA with attenuation of elastic waves increasing tremendously with frequency, the second-order elastic constants increase with frequency as well. ${ }^{36,47}$ It was shown that the second-order elastic constants of PMMA measured by conventional mechanical testing are more than twice smaller than those measured from ultrasonic wave-speeds. In dry sandstones, velocity dispersion is usually considered negligible; ${ }^{48}$ however, it cannot be neglected in fluid saturated sandstones. ${ }^{49}$ In Equations (3) and (4), the second-order elastic constants $\lambda$ and $\mu$ appear multiple times, and they refer either to the quasi-static applied loading or to ultrasonic propagation. Therefore, applying Equations (3) and (4) means that frequency dispersion of the second-order elastic constants is neglected. As a consequence, it is not clear whether these equations can be applied to conventional quasistatic acousto-elastic testing in materials like PMMA. In 
contrast, DAET operates at a few $\mathrm{kHz}$ for the applied strain. Thus, we believe that the estimates of the TOECs obtained with DAET in PMMA are more accurate, because the frequency dispersion of the second-order elastic constants in the $\mathrm{kHz}$ range until $1 \mathrm{MHz}$ (center frequency of the ultrasonic bursts) is small.

\section{Hysteresis}

Berea sandstone is known to exhibit large and complex elastic nonlinearity. ${ }^{27}$ As seen in Figure 3, the relation between the relative change of the ultrasonic wave-speed and the applied strain is not a simple linear relation. Unlike PMMA (Figure 2), we observe hysteresis and DC offset. Their physical origin is still debated. ${ }^{27,50}$ These two features were shown to depend on the amplitude and the frequency of the dynamic loading. ${ }^{33,51}$ However, the first-order derivative (or the slope) of the relation between the relative change of the ultrasonic wave-speed and the loading strain exhibits virtually no dependence on the amplitude and the frequency of the dynamic loading. $^{27,52}$ This fact motivated us to simplify the complicated behavior observed in Figure 3 as a linear relation and derive the TOECs from the slopes of these linear relations.

Hysteresis was also observed when conducting quasistatic acousto-elasticity measurements at moderate stress $(<70 \mathrm{MPa}) .{ }^{53}$ In their work, Winkler et al. ${ }^{16}$ proposed to calculate the first-order derivative of the relation between the wave-speed and the loading strain using the first increasing stress ramp. Why not choosing the decreasing stress ramp? Why not using both increasing and decreasing stress ramps when fitting the model to the experimental data? In our study, a linear regression is applied to the complete nonlinear elastic behavior (increasing strain branch and decreasing strain branch) recorded when cycling the material between a maximal compressive strain of $-8 \times 10^{-6}$ and a maximal tensile strain of $+8 \times 10^{-6}$.

\section{CONCLUSION}

In this work, dynamic acousto-elastic testing (DAET) was applied to the measurement of the three TOECs in PMMA and Berea sandstone. It operates at microstrain about equilibrium state. Therefore, we believe that DAET provides more accurate estimates of the TOECs than conventional quasi-static acousto-elastic experiments, especially in materials exhibiting large elastic nonlinearity like sandstones. The reproducibility of the measurement was studied and taken into account in the calculation of the uncertainty of TOECs estimates. A definite comparison between the DAET and the conventional quasi-static acousto-elastic experiment must be conducted on the very same samples. This subject will be investigated in future work.

\section{APPENDIX A: ON THE CHOICE OF THE INCIDENCE ANGLE AND THE MEASUREMENT CONFIGURATION WITH A SV-WAVE AT OBLIQUE INCIDENCE}

For the measurement configurations with a P-wave at normal incidence and oblique incidence (Figure 1), the ultrasonic signal of interest (that is analyzed with the cross- correlation based method) is the very beginning of the received ultrasonic signal. This is not the case for the measurement configuration with a SV-wave at oblique incidence (Figure 1). In this configuration, the receiving ultrasound transducer records two main signals and the ultrasonic signal of interest is the second one. The first signal arises from the diffraction of the P-wave. The technique based on the crosscorrelation function can only be applied if these two signals do not overlap in time. Therefore, given the diameter $D$ of the cylindrical sample, the temporal duration of an ultrasonic burst $\Delta t$, and the compressional and shear wave-speeds ( $V_{\mathrm{gel}}$, $V_{P}$, and $V_{S}$ ), there can exist a minimal incidence angle $\theta_{\mathrm{gel}}^{M I N}$ so that the two signals do not overlap in time. For a sample with a rather small diameter, if $\frac{D}{\Delta t}\left(1 / V_{S}-1 / V_{P}\right)<1$ then the incidence angle in the coupling gel must exceed the value $\theta_{\text {gel }}^{M I N} \approx \arcsin \left(\frac{V_{\text {gel }}}{V_{S}} \sqrt{\left.1-\left(\frac{D}{\Delta t}\left(1 / V_{S}-1 / V_{P}\right)^{2}\right)\right)}\right.$. In this work, we avoided this problem by using samples with a sufficiently large diameter $\left(\frac{D}{\Delta t}\left(1 / V_{S}-1 / V_{P}\right)>1\right)$.

\section{APPENDIX B: CALCULATION OF THE STRAIN EXPERIENCED BY AN ULTRASONIC PULSE}

The strain experienced by an ultrasonic pulse is calculated from the acceleration measured at one end of the sample and knowing the profile of the first axial compressional resonance mode of the cylindrical sample. ${ }^{30,54}$ The first axial compressional resonance mode is excited when the wavelength equals four times the length of the cylindrical sample. A maximum strain amplitude is obtained at the fixed end of the cylinder while the strain amplitude is null at the free end. ${ }^{30}$ We use samples with a diameter-to-length ratio of 0.15 (i.e., a slenderness ratio of 6.7) for PMMA and 0.17 (i.e., a slenderness ratio of 5.9) for Berea sandstone. As expected, ${ }^{46}$ the observed resonance frequency of the first compressional mode is close to that predicted by the long rod approximation $\frac{1}{4 L} \sqrt{\frac{Y}{\rho}}$, where $L$ is the length of the cylinder, $Y=\frac{\mu(3 \lambda+2 \mu)}{\lambda+\mu}$ is the Young's modulus, and $\rho$ is the mass density. The observed resonance frequency is $1 \%$ smaller than the value predicted by the long rod approximation for PMMA and 3\% smaller for Berea sandstone. Therefore, the applied axial strain $\epsilon_{y y}$ can be assumed constant in the radial dimension, and only the axial modal profile (Figure 1) must be taken into account to estimate the actual strain $\epsilon_{y y}$ experienced by an ultrasonic pulse. ${ }^{30}$

${ }^{1}$ R. Rao and A. Ramanand, Phys. Status Solidi A 58, 11 (1980).

${ }^{2}$ Y. Hiki, Ann. Rev. Mater. Sci. 11, 51 (1981).

${ }^{3}$ A. Norris, "Finite-amplitude waves in solids," in Nonlinear Acoustics (Academic Press, New York, 1998), Chap. 9, pp. 263-277.

${ }^{4}$ G. S. Kino, J. B. Hunter, G. C. Johnson, A. R. Selfridge, D. M. Barnett, G. Hermann, and C. R. Steele, J. Appl. Phys. 50, 2607 (1979).

${ }^{5}$ Y. Pao, W. Sachse, and H. Fukuoka, "Acoustoelasticity and ultrasonic measurements of residual stress," in Physical Acoustics (Academic Press, New York, 1984), Chap. 2, Vol. 17, pp. 61-143.

${ }^{6}$ R. D'Angelo, K. Winkler, T. Plona, B. Landsberger, and D. Johnson, Phys. Rev. Lett. 93, 214301 (2004).

${ }^{7}$ W. Yost and M. Breazeale, J. Appl. Phys. 44, 1909 (1973).

${ }^{8}$ R. A. Graham, J. Acoust. Soc. Am. 51, 1576 (1972).

${ }^{9}$ J. M. Lang and Y. M. Gupta, Phys. Rev. Lett. 106, 125502 (2011). 
${ }^{10}$ R. N. Thurston and K. Brugger, Phys. Rev. A 133, A1604 (1964).

${ }^{11}$ D. Hughes and J. Kelly, Phys. Rev. 92, 1145 (1953).

${ }^{12}$ T. Bateman, W. P. Mason, and H. J. McSkimin, J. Appl. Phys. 32, 928 (1961).

${ }^{13}$ S. Haussühl and W. Chmielewski, Acta Crystallogr., Sect. A 37, 361 (1981).

${ }^{14}$ D. Lazarus, Phys. Rev. 76, 545 (1949).

${ }^{15}$ J. Asay, D. Lamberson, and A. Guenther, J. Appl. Phys. 40, 1768 (1969).

${ }^{16}$ K. Winkler and L. McGowan, J. Geophys. Res. 109, B10204, doi:10.1029/ 2004JB003262 (2004).

${ }^{17}$ P. van't Klooster, N. Trappeniers, and S. Biswas, Physica B + C 97, 65 (1979).

${ }^{18}$ J. K. Krüger, C. Grammes, K. Stockem, R. Zietz, and M. Dettenmaier, Colloid Polym. Sci. 269, 764 (1991).

${ }^{19}$ H. Wang and M. Li, Phys. Rev. B 79, 224102 (2009).

${ }^{20}$ P. Nagy, Ultrasonics 36, 375 (1998).

${ }^{21}$ G. Gremaud, M. Bujard, and W. Benoit, J. Appl. Phys. 61, 1795 (1987).

${ }^{22}$ X. Jacob, C. Christophe Barriere, and D. Royer, Appl. Phys. Lett. 82, 886 (2003).

${ }^{23}$ A. Zeiger and K. Jassby, J. Nondestr. Eval. 3, 115 (1982).

${ }^{24}$ R. Ellwood, T. Stratoudaki, S. D. Sharples, M. Clark, and M. G. Somekh, J. Acoust. Soc. Am. 135, 1064 (2014)

${ }^{25}$ G. Renaud, S. Callé, and M. Defontaine, Appl. Phys. Lett. 94, 011905 (2009).

${ }^{26}$ G. Renaud, J. Rivière, S. Haupert, and P. Laugier, J. Acoust. Soc. Am. 133, 3706 (2013)

${ }^{27}$ J. Rivière, P. Shokouhi, R. Guyer, and P. Johnson, J. Geophys. Res. 120, 1587, doi:10.1002/2014JB011718 (2015).

${ }^{28}$ J. N. Eiras, Q. A. Vu, M. Lott, J. Payá, V. Garnier, and C. Payan, Ultrasonics 69, 29 (2016).

${ }^{29}$ Q. Vu, V. Garnier, J. Chaix, C. Payan, M. Lott, and J. Eiras, Constr. Build. Mater. 114, 87 (2016).

${ }^{30}$ G. Renaud, M. Talmant, S. Callé, M. Defontaine, and P. Laugier, J. Acoust. Soc. Am. 130, 3583 (2011).

${ }^{31}$ G. Renaud, P.-Y. Le Bas, and P. A. Johnson, J. Geophys. Res. 117, B06202, doi:10.1029/2011JB009127 (2012).
${ }^{32}$ B. Mi, J. Michaels, and T. Michaels, J. Acoust. Soc. Am. 119, 74 (2006).

${ }^{33}$ J. Rivière, L. Pimienta, M. Scuderi, T. Candela, P. Shokouhi, J. Fortin, A. Schubnel, C. Marone, and P. Johnson, Geophys. Res. Lett. 43, 3226-3236, doi:10.1002/2016GL068061 (2016).

${ }^{34}$ A. S. Johal and D. J. Dunstan, Phys. Rev. B 73, 024106 (2006).

${ }^{35}$ R. N. Thurston, H. J. McSkimin, and P. Andreatch, J. Appl. Phys. 37, 267 (1966).

${ }^{36}$ M. Batzle, D.-H. Han, and R. Hofmann, Geophysics 71, N1 (2006).

${ }^{37}$ P. A. Johnson and P. N. J. Rasolofosaon, J. Geophys. Res. 101, 3113, doi:10.1029/95JB02880 (1996).

${ }^{38}$ G. Renaud, S. Callé, J.-P. Remenieras, and M. Defontaine, IEEE Trans. UFFC 55(7), 1497 (2008).

${ }^{39}$ D. Egle and D. Bray, J. Acoust. Soc. Am. 60, 741 (1976).

${ }^{40}$ G. J. Meegan, P. Johnson, R. Guyer, and K. McCall, J. Acoust. Soc. Am. 94, 3387 (1993).

${ }^{41}$ J. TenCate, K. Van Den Abeele, T. Shankland, and P. Johnson, J. Acoust. Soc. Am. 100, 1383 (1996).

${ }^{42}$ B. J. Landsberger and M. F. Hamilton, J. Acoust. Soc. Am. 109, 488 (2001).

${ }^{43}$ H. McSkimin and P. J. Andreatch, J. Appl. Phys. 35, 3312 (1964).

${ }^{44}$ R. E. Hankey and D. E. Schuele, J. Acoust. Soc. Am. 48, 190 (1970).

${ }^{45}$ G. Johnson and W. Springer, Int. J. Solids Struct. 25, 609 (1989).

${ }^{46}$ L. Kari, Wave Motion 37, 191 (2003).

${ }^{47}$ J. Capodagli and R. Lakes, Rheol. Acta 47, 777 (2008).

${ }^{48}$ K. Winkler, Geophysics 51, 183 (1986).

${ }^{49}$ L. Pimienta, J. Fortin, and Y. Guéguen, Geophysics 80, D111 (2015).

${ }^{50}$ J. TenCate, Pure Appl. Geophys. 168, 2211 (2011).

${ }^{51}$ K. E. Claytor, J. R. Koby, and J. A. TenCate, Geophys. Res. Lett. 36, L06304, doi:10.1029/2008GL036978 (2009).

${ }^{52}$ G. Renaud, J. Rivière, P.-Y. Le Bas, and P. Johnson, Geophys. Res. Lett. 40, 715, doi:10.1002/grl.50150 (2013).

${ }^{53}$ G. Gist, J. Acoust. Soc. Am. 96, 1158 (1994).

${ }^{54}$ J. Rivière, G. Renaud, R. A. Guyer, and P. A. Johnson, J. Appl. Phys. 114, 054905 (2013). 